\title{
A NEW FIXED POINT THEOREM IN MODULAR METRIC SPACES
}

\author{
ALİ MUTLU ${ }^{1}$, KÜBRA ÖZKAN ${ }^{1, *}$ AND UTKU GÜRDAL ${ }^{2}$ \\ ${ }^{1}$ Manisa Celal Bayar University, Faculty of Science and Arts, Department of Mathematics, 45140, \\ Manisa/TURKEY \\ ${ }^{2}$ Mehmet Akif Ersoy University, Faculty of Science and Arts, Department of Mathematics, \\ Burdur/TURKEY \\ *Corresponding author: kubra.ozkan@hotmail.com
}

\begin{abstract}
In this article, we first give a new fixed point theorem which is main theorem of our study in modular metric spaces. After that, by using this theorem, we express some interesting results. Moreover, we characterize completeness in modular metric spaces via this theorem. Finally, we use our main result to show the existence of solution for a specific problem in dynamic programming.
\end{abstract}

\section{Introduction}

The fixed point theory is used in many different fields of mathematics such as topology, analysis, nonlinear analysis and operator theory. Moreover, it can be applied to different disciplines such as statistics, economy, engineering, etc. In literature, studies of fixed point theory cover a wide range. The most basic and famous fixed point theorem is Banach fixed point theorem which was introduced in 1922 [6]. It guarantees the existence and uniqueness of solution of a functional equation. Besides Banach, many different fixed point theorems were introduced such as Kannan, Caristi, Coupled, Suzuki, etc [1, 2, 7, 8, 13-16, 19, 23, 24].

In 1950, Nakano introduced modular spaces [21]. Then Chistyakov introduced the concept of modular metric spaces, which have a physical interpretation, via F-modulars [9] in 2008 and he further developed

Received 2018-03-20; accepted 2018-05-09; published 2018-07-02.

2010 Mathematics Subject Classification. 46A80, 47H10, 54H25.

Key words and phrases. modular metric space; fixed point theorem; complete modular metric.

(c)2018 Authors retain the copyrights of their papers, and all open access articles are distributed under the terms of the Creative Commons Attribution License. 
the theory of these spaces in 2010 [10]. Then many authors made various studies on this structures, e.g. $[3-5,11,12,17,18,20]$.

In this paper, we first give a new fixed point theorem which is main theorem of our study. After that, by using this theorem, we express some interesting results. Moreover, we characterize completeness in modular metric spaces via this theorem. Finally, we use our main theorem to show the existence of solution for a specific problem in dynamic programming.

\section{Modular Metric Spaces}

Here, we express a series of definitions of some basic concepts related to modular metric spaces.

Definition 2.1. [22] Let $X$ be a linear space on $\mathbb{R}$. If a functional $\rho: X \rightarrow[0, \infty]$ satisfies the following conditions, we call that $\rho$ is a modular on $X$ :

(1) $\rho(0)=0$;

(2) If $x \in X$ and $\rho(\alpha x)=0$ for all numbers $\alpha>0$, then $x=0$;

(3) $\rho(-x)=\rho(x)$, for all $x \in X$;

(4) $\rho(\alpha x+\beta y) \leq \rho(x)+\rho(y)$ for all $\alpha, \beta \geq 0$ with $\alpha+\beta=1$ and $x, y \in X$.

Let $X \neq \emptyset$ and $\lambda \in(0, \infty)$. Generally, a function $\omega:(0, \infty) \times X \times X \rightarrow[0, \infty]$ is denoted as $\omega_{\lambda}(x, y)=$ $\omega(\lambda, x, y)$ for all $x, y \in X$ and $\lambda>0$.

Definition 2.2. [10] Let $X \neq \emptyset$. A function $\omega:(0, \infty) \times X \times X \rightarrow[0, \infty]$, which satisfies the following conditions for all $x, y, z \in X$, is called a metric modular on $X$ :

(m1) $\omega_{\lambda}(x, y)=0$ for all $\lambda>0 \Leftrightarrow x=y$;

(m2) $\omega_{\lambda}(x, y)=\omega_{\lambda}(y, x)$ for all $\lambda>0$;

(m3) $\omega_{\lambda+\mu}(x, y) \leq \omega_{\lambda}(x, z)+\omega_{\mu}(z, y)$ for all $\lambda, \mu>0$.

If $0<\mu<\lambda$, from properties of metric modular, we obtain that

$$
\omega_{\lambda}(x, y) \leq \omega_{\lambda-\mu}(x, x)+\omega_{\mu}(x, y)=\omega_{\mu}(x, y)
$$

for all $x, y \in X$.

From $[10,11]$, we know that for a fixed $x_{0} \in X$, the two sets

$$
X_{\omega}=X_{\omega}\left(x_{0}\right)=\left\{x \in X: \omega_{\lambda}\left(x, x_{0}\right) \rightarrow 0 \text { as } \lambda \rightarrow \infty\right\}
$$

and

$$
X_{\omega}^{*}=X_{\omega}^{*}\left(x_{0}\right)=\left\{x \in X: \exists \lambda=\lambda(x)>0 \text { such that } \omega_{\lambda}\left(x, x_{0}\right)<\infty\right\}
$$

are said to be modular spaces. 
It is known $[10,11]$ that if $\omega$ is a metric modular on a nonempty set $X$, then the modular space $X_{\omega}$ can be equipped with a metric, generated by $\omega$ and given by

$$
d_{\omega}(x, y)=\inf \left\{\lambda>0: \omega_{\lambda}(x, y) \leq \lambda\right\}
$$

for all $x, y \in X_{\omega}$. The pair $\left(X_{\omega}, d_{\omega}\right)$ is called a modular metric space.

Definition 2.3. [18] Let $X_{\omega}$ be a modular metric space, $\left\{x_{n}\right\}_{n \in \mathbb{N}}$ be a sequence in $X_{\omega}$ and $C \subseteq X_{\omega}$. Then (1) $\left\{x_{n}\right\}_{n \in \mathbb{N}}$ is called a modular convergent sequence such that $x_{n} \rightarrow x, x \in X_{\omega}$, if for $\lambda>0$

$$
\omega_{\lambda}\left(x_{n}, x\right) \rightarrow 0 \text { as } n \rightarrow \infty
$$

(2) $\left\{x_{n}\right\}_{n \in \mathbb{N}}$ is called a modular Cauchy sequence, if for $\lambda>0$

$$
\omega_{\lambda}\left(x_{n}, x_{m}\right) \rightarrow 0 \text { as } m, n \rightarrow \infty
$$

(3) $C$ is called closed, if the limit of a modular convergent sequence in $C$ always belongs to $C$.

(4) $C$ is called complete modular, if every modular Cauchy sequence $\left\{x_{n}\right\}$ in $C$ is modular convergent in $C$.

(5) $C$ is called bounded, if

$$
\delta_{\omega}(C)=\sup \left\{\omega_{\lambda}(x, y): x, y \in C, \lambda>0\right\}<\infty .
$$

\section{Main Results}

Let $\omega:(0, \infty) \times X \times X \rightarrow[0, \infty]$ be a metric modular on $X, X_{\omega}$ be a modular metric space, $C \subseteq X_{\omega}$ and $\psi: C \rightarrow \mathbb{R}^{+}$be a function on $C$. $\psi$ is called lower semi-continuous (l.s.c.) on $C$ if

$$
\lim _{n \rightarrow \infty} \omega_{\lambda}\left(x_{n}, x\right)=0 \Rightarrow \psi(x) \leq \lim _{n \rightarrow \infty} \inf \left(\psi\left(x_{n}\right)\right)
$$

for all $\left\{x_{n}\right\} \subseteq C$ and $\lambda>0$.

Theorem 3.1. Let $\omega$ be a metric modular on $X, X_{\omega}$ be a complete modular metric space, $\psi: X_{\omega} \rightarrow \mathbb{R}^{+}$be a lower semi-continuous function on $X_{\omega}$ and $T: X_{\omega} \rightarrow X_{\omega}$ be a mapping satisfying the inequality

$$
\omega_{\lambda}(x, T x) \leq \psi(x)-\psi(T x)
$$

for all $x \in X_{\omega}$ and $\lambda>0$. Then $T$ has a fixed point in $X_{\omega}$.

Proof. For any $x \in X_{\omega}$ denote,

$$
\begin{aligned}
& P(x)=\left\{y \in X_{\omega}: \omega_{\lambda}(x, y) \leq \psi(x)-\psi(y) \text { for all } \lambda>0\right\} \\
& \alpha(x)=\inf \{\psi(y): y \in P(x)\}
\end{aligned}
$$


As $x \in P(x), P(x)$ is not empty and $0 \leq \alpha(x) \leq \psi(x)$. Let $x \in X_{\omega}$ be an arbitrary point. Now, we construct a sequence $\left\{x_{n}\right\}$ in $X_{\omega}$ as follows: Let $x_{1}=x$ and when $x_{1}, x_{2}, \ldots, x_{n}$ have been chosen, choose $x_{n+1} \in P\left(x_{n}\right)$ such that $\psi\left(x_{n+1}\right) \leq \alpha\left(x_{n}\right)+\frac{1}{n}$, for all $n \in \mathbb{N}$. By doing so, we get a sequence $\left\{x_{n}\right\}$ satisfying the conditions

$$
\begin{aligned}
\omega_{\lambda}\left(x_{n}, x_{n+1}\right) & \leq \psi\left(x_{n}\right)-\psi\left(x_{n+1}\right), \\
\alpha\left(x_{n}\right) & \leq \psi\left(x_{n+1}\right) \leq \alpha\left(x_{n}\right)+\frac{1}{n}
\end{aligned}
$$

for all $n \in \mathbb{N}$ and $\lambda>0$. Then $\left\{\psi\left(x_{n}\right)\right\}$ is a nonincreasing sequence in $\mathbb{R}$ and it is bounded from below by zero. So, the sequence $\left\{\psi\left(x_{n}\right)\right\}$ is convergent to a number $M \geq 0$. By virtue of (3.2), we get

$$
M=\lim _{n \rightarrow \infty} \psi\left(x_{n}\right)=\lim _{n \rightarrow \infty} \alpha\left(x_{n}\right) .
$$

Now, let $k \in \mathbb{N}$ be arbitrary. From (3.2) and (3.3), there exists at least a number $N_{k}$ such that $\psi\left(x_{n}\right)<M+\frac{1}{k}$ for all $n \geq N_{k}$. Since $\psi\left(x_{n}\right)$ is monotone, we get

$$
M \leq \psi\left(x_{m}\right) \leq \psi\left(x_{n}\right)<M+\frac{1}{k}
$$

for $m \geq n \geq N_{k}$. It follows that

$$
\psi\left(x_{n}\right)-\psi\left(x_{m}\right)<\frac{1}{k} \quad \text { for all } \quad m \geq n \geq N_{k} .
$$

Preserving the generality, suppose that $m>n$ and $m, n \in \mathbb{N}$. From (3.2), we get

$$
\omega_{\frac{\lambda}{m-n}}\left(x_{n}, x_{n+1}\right) \leq \psi\left(x_{n}\right)-\psi\left(x_{n+1}\right)
$$

for $\frac{\lambda}{m-n}>0$. Now, we obtain that

$$
\begin{aligned}
\omega_{\lambda}\left(x_{n}, x_{m}\right) & \leq \omega_{\frac{\lambda}{m-n}}\left(x_{n}, x_{n+1}\right)+\omega_{\frac{\lambda}{m-n}}\left(x_{n+1}, x_{n+2}\right)+\cdots+\omega_{\frac{\lambda}{m-n}}\left(x_{m-1}, x_{m}\right) \\
& \leq \psi\left(x_{n}\right)-\psi\left(x_{n+1}\right)+\psi\left(x_{n+1}\right)-\psi\left(x_{n+2}\right)+\cdots+\psi\left(x_{m-1}\right)-\psi\left(x_{m}\right) \\
& =\psi\left(x_{n}\right)-\psi\left(x_{m}\right)
\end{aligned}
$$

for all $m, n \geq N_{k}$. Then by (3.4),

$$
\omega_{\lambda}\left(x_{n}, x_{m}\right)<\frac{1}{k} \quad \text { for all } \quad m \geq n \geq N_{k} .
$$

Letting $k$ or $m$ and $n$ tend to infinity in (3.6), we conclude that

$$
\lim _{m, n \rightarrow \infty} \omega_{\lambda}\left(x_{n}, x_{m}\right)=0 .
$$

Then $\left\{x_{n}\right\}_{n \in \mathbb{N}}$ is a modular Cauchy sequence. Since $X_{\omega}$ is complete modular, there exists a point $u \in X_{\omega}$ such that $x_{n} \rightarrow u$ as $n \rightarrow \infty$. Since $\psi$ is lower semi-continuous, using (3.5), we have

$$
\begin{aligned}
\psi(u) & \leq \lim _{m \rightarrow \infty} \inf \psi\left(x_{m}\right) \\
& \leq \lim _{m \rightarrow \infty} \inf \left(\psi\left(x_{n}\right)-\omega_{\lambda}\left(x_{n}, x_{m}\right)\right) \\
& =\psi\left(x_{n}\right)-\omega_{\lambda}\left(x_{n}, u\right)
\end{aligned}
$$


and hence

$$
\omega_{\lambda}\left(x_{n}, u\right) \leq \psi\left(x_{n}\right)-\psi(u)
$$

Thus, $u \in P\left(x_{n}\right)$ for all $n \in \mathbb{N}$ and hence $\alpha\left(x_{n}\right) \leq \psi(u)$. Then by (3.3), we get $M \leq \psi(u)$. Moreover, using lower semi-continuity of $\psi$ and (3.3), we have

$$
\psi(u) \leq \lim _{n \rightarrow \infty} \inf \psi\left(x_{n}\right)=M
$$

So, $\psi(u)=M$. From (3.1), we know that $T u \in P(u)$. Since $u \in P(u)$ for $n \in \mathbb{N}$, we have

$$
\begin{aligned}
\omega_{\lambda}\left(x_{n}, T u\right) & \leq \omega_{\frac{\lambda}{2}}\left(x_{n}, u\right)+\omega_{\frac{\lambda}{2}}(u, T u) \\
& \leq \psi\left(x_{n}\right)-\psi(u)+\psi(u)-\psi(T u) \\
& =\psi\left(x_{n}\right)-\psi(T u) .
\end{aligned}
$$

Then $T u \in P\left(x_{n}\right)$ and this implies $\alpha\left(x_{n}\right) \leq \psi(T u)$. Hence, we obtain $M \leq \psi(T u)$. From (3.1), we get $\psi(T u) \leq \psi(u)$. As $\psi(u)=M$, we have

$$
\psi(u)=M \leq \psi(T u) \leq \psi(u) .
$$

Therefore, $\psi(T u)=\psi(u)$. Then from (3.1), we get

$$
\omega_{\lambda}(u, T u) \leq \psi(u)-\psi(T u)=0 .
$$

Thus, $T u=u$.

Theorem 3.2. Let $\omega$ be a metric modular on $X$ and $X_{\omega}$ be a complete modular metric space and $\psi: X_{\omega} \rightarrow \mathbb{R}$ be a lower semi-continuous function on $X_{\omega}$. If $\psi$ is bounded below, then there exists a point $u \in X_{\omega}$ such that

$$
\psi(u)<\psi(x)+\omega_{\lambda}(u, x)
$$

for each $x \in X_{\omega}, x \neq u$ and $\lambda>0$.

Proof. Following the proof Theorem 3.1, we obtain a sequence $\left\{x_{n}\right\}$ that converges to some $u \in X_{\omega}$. Under the same notations, for any $u \in X_{\omega}$, define

$$
\begin{aligned}
& P(u)=\left\{x \in X_{\omega}: \omega_{\lambda}(u, x) \leq \psi(u)-\psi(x) \text { for all } \lambda>0\right\} \\
& \alpha(u)=\inf \{\psi(x): x \in P(u)\} .
\end{aligned}
$$


We will show that $u \notin P(u)$ as $x \neq u$. Suppose the contrary, that is, we get $v \in P(u)$ for some $v \neq u$. Then $0<\omega_{\lambda}(u, v) \leq \psi(u)-\psi(v)$ implies $\psi(v)<\psi(u)=M$. Since

$$
\begin{aligned}
\omega_{\lambda}\left(x_{n}, v\right) & \leq \omega_{\frac{\lambda}{2}}\left(x_{n}, u\right)+\omega_{\frac{\lambda}{2}}(u, v) \\
& \leq \psi\left(x_{n}\right)-\psi(u)+\psi(u)-\psi(v) \\
& =\psi\left(x_{n}\right)-\psi(v)
\end{aligned}
$$

for all $\lambda>0, v \in P\left(x_{n}\right)$. So,

$$
\alpha\left(x_{n}\right) \leq \psi(v) \text { for all } n \in \mathbb{N}
$$

Letting $n$ tends to infinity, we get $M \leq \psi(v)$. This equation contradicts with $\psi(v)<M=\psi(u)$. Therefore, for each $x \in X_{\omega}, x \neq u$ implies $x \notin P(u)$, that is

$$
x \neq u \Rightarrow \omega_{\lambda}(u, x)>\psi(u)-\psi(x) .
$$

Theorem 3.3. Let $X_{\omega}$ and $Y_{\omega}$ be complete modular metric spaces and the mapping $T: X_{\omega} \rightarrow X_{\omega}$ be arbitrary. Assume that there exists a closed mapping $S: X_{\omega} \rightarrow Y_{\omega}$, a lower semi-continuous mapping $\psi: S\left(X_{\omega}\right) \rightarrow \mathbb{R}^{+}$and a constant $c>0$ such that for any $x \in X_{\omega}$ and $\lambda>0$

$$
\begin{aligned}
\omega_{\lambda}(x, T x) & \leq \psi(S x)-\psi(S T x) \text { and } \\
\left.c \cdot \omega_{\lambda}(S x, S T x)\right\} & \leq \psi(S x)-\psi(S T x) .
\end{aligned}
$$

Then the mapping $T$ has a fixed point.

Proof. For any $x \in X_{\omega}$, we set

$$
\begin{aligned}
P(x)= & \left\{z \in X_{\omega}: \omega_{\lambda}(x, z) \leq \psi(S x)-\psi(S z)\right. \text { and } \\
& \left.\left.c \cdot \omega_{\lambda}(S x, S z)\right\} \leq \psi(S x)-\psi(S z) \text { for all } \lambda>0\right\} \\
\alpha(x)= & \inf \{\psi(S z): z \in P(x)\} .
\end{aligned}
$$

As $x \in P(x)$, it is clear that $P(x) \neq \emptyset$ and $0 \leq \alpha(x) \leq \psi(S x)$. Similar to the proof of Theorem 3.1, choose a sequence $\left\{x_{n}\right\}$ in $X_{\omega}: x_{1}=x, x_{n+1} \in P\left(x_{n}\right)$ such that

$$
\psi\left(S x_{n+1}\right) \leq \alpha\left(x_{n}\right)+\frac{1}{n}
$$

for all $n \geq 1$. Thus we obtain that

$$
\begin{aligned}
\omega_{\lambda}\left(x_{n}, x_{n+1}\right) & \leq \psi\left(S x_{n}\right)-\psi\left(S x_{n+1}\right), \\
\left.c \cdot \omega_{\lambda}\left(S x_{n}, S x_{n+1}\right)\right\} & \leq \psi\left(S x_{n}\right)-\psi\left(S x_{n+1}\right)
\end{aligned}
$$


and

$$
\psi\left(S x_{n+1}\right)-\frac{1}{n} \leq \alpha\left(x_{n}\right) \leq \psi\left(S x_{n+1}\right) .
$$

From (3.8), $\left\{\psi\left(S x_{n}\right)\right\}$ is a nonincreasing and bounded sequence on $\mathbb{R}$. So, $\left\{\psi\left(S x_{n}\right)\right\}$ is a modular convergent sequence. Therefore, by (3.9) there is a number $M \geq 0$ such that

$$
M=\lim _{n \rightarrow \infty} \alpha\left(x_{n}\right)=\lim _{n \rightarrow \infty} \psi\left(S x_{n}\right) .
$$

Now, let $k \in \mathbb{N}$ be an arbitrary point. From (3.10), there exists some $N_{k}$ such that $\psi\left(S x_{n}\right)<M+\frac{1}{k}$ for all $n \geq N_{k}$. Thus, by monotonicity of $\left\{\psi\left(S x_{n}\right)\right\}$, for all $m \geq n \geq N_{k}$ we have

$$
M \leq \psi\left(S x_{m}\right) \leq \psi\left(S x_{n}\right) \leq M+\frac{1}{k} .
$$

So,

$$
\psi\left(S x_{n}\right)-\psi\left(S x_{m}\right) \leq \frac{1}{k}
$$

Preserving the generality, suppose that $m>n$ and $m, n \in \mathbb{N}$. From (3.8), we easily obtain that

$$
\begin{aligned}
\omega_{\frac{\lambda}{m-n}}\left(x_{n}, x_{n+1}\right) & \leq \psi\left(S x_{n}\right)-\psi\left(S x_{n+1}\right) \text { and } \\
\left.c \cdot \omega_{\frac{\lambda}{m-n}}\left(S x_{n}, S T x_{n+1}\right)\right\} & \leq \psi\left(S x_{n}\right)-\psi\left(S x_{n+1}\right)
\end{aligned}
$$

for $\frac{\lambda}{m-n}>0$. From (3.8), (3.12) and condition (M3) of modular metric, we have

$$
\begin{aligned}
\omega_{\lambda}\left(x_{n}, x_{m}\right) \leq & \omega_{\frac{\lambda}{m-n}}\left(x_{n}, x_{n+1}\right)+\omega_{\frac{\lambda}{m-n}}\left(x_{n+1}, x_{n+2}\right)+\cdots+\omega_{\frac{\lambda}{m-n}}\left(x_{m-1}, x_{m}\right) \\
\leq & \psi\left(S x_{n}\right)-\psi\left(S x_{n+1}\right)+\psi\left(S x_{n+1}\right)-\psi\left(S x_{n+2}\right) \\
& +\cdots+\psi\left(S x_{m-1}\right)-\psi\left(S x_{m}\right) \\
= & \psi\left(S x_{n}\right)-\psi\left(S x_{m}\right) \\
c \cdot \omega_{\lambda}\left(S x_{n}, S x_{m}\right) \leq & c \cdot \omega_{\frac{\lambda}{m-n}}\left(S x_{n}, S x_{n+1}\right)+c \cdot \omega_{\frac{\lambda}{m-n}}\left(S x_{n+1}, S x_{n+2}\right) \\
& +\cdots+c \omega_{\frac{\lambda}{m-n}}\left(S x_{m-1}, S x_{m}\right) \\
\leq & \psi\left(S x_{n}\right)-\psi\left(S x_{n+1}\right)+\psi\left(S x_{n+1}\right)-\psi\left(S x_{n+2}\right) \\
& +\cdots+\psi\left(S x_{m-1}\right)-\psi\left(S x_{m}\right) \\
= & \psi\left(S x_{n}\right)-\psi\left(S x_{m}\right) .
\end{aligned}
$$

From (3.11), we get

$$
\omega_{\lambda}\left(x_{n}, x_{m}\right)<\frac{1}{k} \text { and } c \cdot \omega_{\lambda}\left(S x_{n}, S x_{m}\right)<\frac{1}{k}
$$

for all $m \leq n \leq N_{k}$ and $k \in \mathbb{N}$. Therefore, $\left\{x_{n}\right\}_{n \in \mathbb{N}}$ is a modular Cauchy sequence in $X_{\omega}$ and $\left\{S x_{n}\right\}_{n \in \mathbb{N}}$ is a modular Cauchy sequence in $Y_{\omega}$. By completeness of $X_{\omega}$ and $Y_{\omega}$, there exist $p \in X_{\omega}$ and $q \in Y_{\omega}$ such that 
$x_{n} \rightarrow p$ and $S x_{n} \rightarrow q$. The fact that, $S$ is a closed mapping implies $S p=q$. Since $\psi$ is lower semi-continuous, using equation (3.13), we have

$$
\begin{aligned}
\psi(q)=\psi(S p) \leq \lim _{m \rightarrow \infty} \inf \psi\left(S x_{m}\right) & \leq \lim _{m \rightarrow \infty} \inf \left(\psi\left(S x_{n}\right)-\omega_{\lambda}\left(x_{n}, x_{m}\right)\right) \\
& =\psi\left(S x_{n}\right)-\omega_{\lambda}\left(x_{n}, p\right)
\end{aligned}
$$

Then we obtain

$$
\omega_{\lambda}\left(x_{n}, p\right) \leq \psi\left(S x_{n}\right)-\psi(S p)
$$

for $\lambda>0$. Similarly, we get

$$
c \cdot \omega_{\lambda}\left(x_{n}, p\right) \leq \psi\left(S x_{n}\right)-\psi(S p)
$$

Thus, $p \in P\left(x_{n}\right)$ for all $n \in \mathbb{N}$. Then $\alpha\left(x_{n}\right) \leq \psi(S p)$. So, by (3.10), we get $M \leq \psi(S p)$. On the other hand, using lower semi-continuity of $\psi$ and (3.10), we have

$$
\psi(q)=\psi(S p)=\lim _{m \rightarrow \infty} \alpha\left(x_{n}\right)=M
$$

Therefore, $\psi(S p)=M$. By benefiting from the proof of Theorem 3.2, we obtain that $x \neq p$ implies $x \notin P(p)$. From (3.7), it's clear that $T p \in P(p)$, then we have $T p=p$.

Corollary 3.1. Theorem 3.3 holds with inequality

$$
\max \left\{\omega_{\lambda}(x, T x), c \cdot \omega_{\lambda}(S x, S T x)\right\} \leq \psi(S x)-\psi(S T x)
$$

in the place of inequality (3.7).

Example 3.1. Let $X=\mathbb{R}$. We define the mapping $\omega:(0, \infty) \times \mathbb{R} \times \mathbb{R} \rightarrow[0, \infty]$ by $\omega_{\lambda}(x, y)=\frac{|x-y|}{1+\lambda}$ for all $x, y \in \mathbb{R}$ and $\lambda>0$. Then it is obvious that $\mathbb{R}_{\omega}$ is a complete modular metric space. Define $T: \mathbb{R}_{\omega} \rightarrow \mathbb{R}_{\omega}$ by $T x=\frac{x}{4}$ and $\psi: \mathbb{R}_{\omega} \rightarrow[0, \infty]$ by $\psi(x)=3|x|$. Then for all $x, y \in \mathbb{R}$ and $\lambda>0$, we have

$$
\omega_{\lambda}(x, T x)=\frac{|x-T x|}{1+\lambda}=\frac{\left|x-\frac{x}{4}\right|}{1+\lambda}=\frac{3|x|}{4(1+\lambda)} \leq \frac{3}{4}|x|
$$

and

$$
\psi(x)-\psi(T x)=3|x|-\frac{3|x|}{4}=\frac{9}{4}|x|
$$

Hence, $\omega_{\lambda}(x, T x) \leq \psi(x)-\psi(T x)$. From Theorem 3.1, the mapping $T$ has a fixed point. Moreover, it is $0 \in \mathbb{R}_{\omega}$. 


\section{Characterization of Completeness}

We now prove a new theorem, which together with Theorem 3.1 characterizes completeness in modular metric spaces.

Theorem 4.1. Let $X_{\omega}$ be a modular metric space which is not complete modular. Then there exists a fixed point free function $T: X_{\omega} \rightarrow X_{\omega}$ and a lower semi-continuous mapping $\psi: X_{\omega} \rightarrow \mathbb{R}^{+}$such that

$$
\omega_{\lambda}(x, T x) \leq \psi(x)-\psi(T x)
$$

for all $x \in X_{\omega}$ and $\lambda>0$.

Proof. Let $\left\{x_{n}\right\} \subset X_{\omega}$ be a modular Cauchy sequence, which has no limit. We define a function $\phi: X_{\omega} \rightarrow \mathbb{R}^{+}$ by

$$
\phi(u)=\lim _{m \rightarrow \infty} \omega_{\lambda}\left(u, x_{m}\right), u \in X_{\omega} \text { for all } \lambda>0 .
$$

Given $x \in X_{\omega}$ and let $n$ denote the smallest positive integer such that

$$
0<\frac{1}{2} \omega_{\lambda}\left(x, x_{n}\right) \leq \phi(x)-\phi\left(x_{n}\right) \text { for all } \lambda>0
$$

Note that $\phi\left(x_{n}\right) \rightarrow 0$ as $\phi(x)>0$. With $n$ so determined, we define function $T: X_{\omega} \rightarrow X_{\omega}$ as $T x=x_{n}$. Let $\psi(x)=2 \phi(x)$. Then from (4.1), we obtain that

$$
\omega_{\lambda}(x, T x) \leq \psi(x)-\psi(T x)
$$

\section{Application}

Let $X_{\omega}$ be a complete modular metric space, $Y$ be a Banach space, $M \subseteq X_{\omega}, S \subseteq Y$ and $\theta: M \times S \rightarrow M$, $H: M \times S \times \mathbb{R} \rightarrow \mathbb{R}$ be two functions. Using Theorem 3.1, we show the existence of a bounded solution for the following problem in dynamic programming:

We take a $g \in B(M)$ such that

$$
g(t)=\sup _{s \in S}\{H(t, s, g(\theta(t, s)))\}
$$

where $t \in M$ and $B(M)$ is a Banach space which consists of all bounded real functionals on $M$ with the norm $\|g\|=\sup _{t \in M}|g(t)|$. We define a complete modular metric on $B(M)$ with

$$
\omega_{\lambda}(g, k)=\sup _{t \in Z}\left\{\left|\frac{g(t)-k(t)}{1+\lambda}\right|\right\}
$$

for all $g, k \in B(M)$ and $\lambda>0$. If we take a Cauchy sequence $\left\{g_{n}\right\}_{n \in \mathbb{N}}$ in $B(M)$, then from completeness of $X_{\omega}$, there exists a function $u \in B(M)$ such that the sequence $\left\{g_{n}\right\}_{n \in \mathbb{N}}$ is convergent to $u$. 
Theorem 5.1. Let $\theta: M \times S \rightarrow M, H: M \times S \times \mathbb{R} \rightarrow \mathbb{R}$ be bounded and $\psi: B(M) \rightarrow \mathbb{R}^{+}$be lower semi continuous on $X_{\omega}$ and define by $\psi(g)=\|g\|$. We define a operator $T: B(M) \rightarrow B(M)$ by

$$
T(g)(t)=\sup _{s \in S}\{H(t, s, g(\theta(t, s)))\}
$$

for all $g \in B(M)$ and $t \in M$. If

$$
\sup _{t \in M}\left|\frac{g(t)-H(t, s, g(\theta(t, s)))}{\lambda}\right| \leq \psi(g)-\psi(T(g))
$$

for all $\lambda>0, g, k \in B(M)$ and $s \in S$, then the functional equation (5.1) has a bounded solution.

Proof. Let $t \in M$ and $g \in B(M)$. Then there exists $s \in S$ and $\epsilon>0$ such that

$$
T(g)(x)<H(t, s, g(\theta(t, s)))+\epsilon
$$

and

$$
T(g)(x)>H(t, s, g(\theta(t, s))) .
$$

On the other hand, it is obvious that

$$
g(t)<g(t)+\epsilon
$$

and

$$
g(t)>g(t)-\epsilon
$$

for all $\epsilon>0$. By using the inequalities (5.5) and (5.6) we obtain that

$$
\begin{aligned}
g(t)-T(g)(t) & <g(t)-H(t, s, g(\theta(t, s)))+\epsilon \\
& \leq|g(t)-H(t, s, g(\theta(t, s)))|+\epsilon .
\end{aligned}
$$

Similarly, by using the inequalities (5.4) and (5.7) we obtain that

$$
\begin{aligned}
T(g)(t)-g(t) & <H(t, s, g(\theta(t, s)))-g(t)+2 \epsilon \\
& \leq|H(t, s, g(\theta(t, s)))-g(t)|+2 \epsilon .
\end{aligned}
$$

Therefore, from the inequalities (5.8) and (5.9), we get

$$
|g(t)-T(g)(t)|<|g(t)-H(t, s, g(\theta(t, s)))|+2 \epsilon .
$$

If we divide both sides of the inequality (5.10) by $1+\lambda$, we get

$$
\left|\frac{g(t)-T(g)(t)}{1+\lambda}\right|<\left|\frac{g(t)-H(t, s, g(\theta(t, s)))}{1+\lambda}\right|+\frac{2 \epsilon}{1+\lambda}
$$

for all $\lambda>0$. Since $\frac{2 \epsilon}{1+\lambda}>0$ in the inequality (5.11), we can ignore the contrary $\frac{2 \epsilon}{1+\lambda}$. Then we have

$$
\left|\frac{g(t)-T(g)(t)}{1+\lambda}\right|<\left|\frac{g(t)-H(t, s, g(\theta(t, s)))}{1+\lambda}\right|
$$


for all $\lambda>0$. Then from property of supremum, we get

$$
\sup _{t \in Z}\left|\frac{g(t)-T(g)(t)}{1+\lambda}\right|<\sup _{t \in Z}\left|\frac{g(t)-H(t, s, g(\theta(t, s)))}{1+\lambda}\right| .
$$

Then from inequalities (5.2) and (5.3) we obtain that

$$
\omega_{\lambda}(g, T(g))<\psi(g)-\psi(T(g))
$$

Therefore, from Theorem 3.1, $T$ has a fixed point $u \in B(Z)$. Then the functional equation (5.1) has a bounded solution.

\section{REFERENCES}

[1] Ö. Acar and I. Altun, Some generalizations of Caristi type fixed point theorem on partial metric spaces, Filomat, 26(4) (2012), 833-837.

[2] H. Aydi and M. Abbas, Tripled coincidence and fixed point results in partial metric spaces, Appl. Gen. Topol., 13(2) (2012), 193-206.

[3] A.A.N. Abdou and M.A. Khamsi, Fixed point results of pointwise contractions in modular metric spaces, Fixed Point Theory Appl., 2013 (2013), Article ID 163.

[4] A.A.N. Abdou and M.A. Khamsi, Fixed points of multivalued contraction mappings in modular metric spaces, Fixed Point Theory Appl., 2014 (2014), Article ID 249.

[5] C. Alaca, M.E. Ege and C. Park, Fixed point results for modular ultrametric spaces, J. Comput. Anal. Appl., 20(1) (2016), 1259-1267.

[6] S. Banach, Sur les opérations dans les ensembles abstraits et leur application aux équations intégrales, Fund. Math., 3 (1922), 133-181.

[7] T.G. Bhaskar and V. Lakshmikantham, Fixed point theorems in partially ordered metric spaces and applications, Nonlinear Anal., 65 (2006), 1379-1393.

[8] J. Caristi, Fixed point theorems for mappings satisfying inwardness conditions, Trans. Am. Math. Soc., 215 (1976), $241-251$.

[9] V.V. Chistyakov, Modular metric spaces generated by F-modulars, Folia Math., 14 (2008), 3-25.

[10] V.V. Chistyakov, Modular metric spaces, I: basic concepts, Nonlinear Anal., 72 (2010), 1-14.

[11] V.V. Chistyakov, Fixed points of modular contractive maps, Doklady Math., 86(1) (2012), 515-518.

[12] Y.J. Cho, R. Saadati and G. Sadeghi, Quasi-contractive mappings in modular metric spaces, J. Appl. Math., 2012 (2012), 907-951.

[13] I. Erhan, E. Karapinar and D. Turkoglu, Different types Meir-Keeler contractions on partial metric, J. Comput. Anal. Appl, 14 (2012), 1000-1005.

[14] R. Kannan, Some results on fixed points, Bull. Calcutta Math. Soc., 60 (1968), 71-76.

[15] M.A. Khamsi, Remarks on Caristi's fixed point theorem, Nonlinear Anal., 71(1-2) (2009), 227-231.

[16] W.A. Kirk, Caristi's fixed point theorem and metric convexity, Colloq. Math., 36 (1976), 81-86.

[17] P. Kumam, Fixed point theorems for nonexpansive mapping in modular spaces, Arch. Math., 40 (2004), $345-353$.

[18] C. Mongkolkeha, W. Sintunavarat and P. Kumam, Fixed point theorems for contraction mappings in modular metric spaces, Fixed Point Theory Appl., 2011 (2011), Article ID 93.

[19] A. Mutlu, K. Özkan and U. Gürdal, Coupled fixed point theorems on bipolar metric spaces, Eur. J. Pure Appl. Math., 10(4) (2017), 655-667. 
[20] A. Mutlu, K. Özkan and U. Gürdal, Coupled fixed point theorem in partially ordered modular metric spaces and its an application, J. Comput. Anal. Appl., 25(2) (2018), 1-10.

[21] H. Nakano, Modulared Semi-Ordered Linear Spaces, In Tokyo Math. Book Ser., 1, Maruzen Co. Tokyo, 1950.

[22] J. Musielak and W. Orlicz, On modular spaces, Studia Math., 18 (1959), 49-65.

[23] W. Shatanawi, B. Samet and M. Abbas, Coupled fixed point theorems for mixed monotone mappings in ordered partial metric spaces, Math. Comput. Model., 55(3-4) (2012), 680-687.

[24] T. Suzuki, A new type of fixed point theorem in metric spaces, Nonlinear Anal., 71 (2009), 5313-5317. 